\title{
An assessment of the Kemble Star 700 sample processor* for the automation of complement fixation and ELISA tests in a microbiology laboratory
}

\author{
M. AMPHLETT, D. J. SMITH and R. E. WARREN \\ Clinical Microbiology and Public Health Laboratory, Addenbrookes Hospital, Cambridge CB2 $20 Q$
}

\begin{abstract}
Summary. A Kemble Robotic sample processor was programmed to perform routine serological tests for microbiology. Accuracy of dispensed volumes was assessed and precision of dilutions was compared with manual methods. An appraisal of time taken to perform complement-fixation and enzyme-linked immunosorbent assays by manual and automated methods was undertaken. Labour requirements were reduced when processing large numbers of samples and the reproducibility of more demanding tests was improved by the greater accuracy of the robotic manipulations.
\end{abstract}

\section{Introduction}

In the methods originally described for complementfixation tests (CFTs) and enzyme-linked immunosorbent assays (ELISA) dilutions were done in test tubes; they were then modified and adapted to microtitration plates. In our laboratory, CFTs have been performed routinely with an eight-channel peristaltic pump with a hand set (Microcompupet) which enables liquid to be dispensed and diluted simultaneously in eight rows of a microtitration plate. This requires the continuous presence of technical staff. ELISA techniques have not been mechanised in this way and are performed with reagents dispensed by micropipette.

Difficulties in the recruitment, training and retention of skilled staff, together with the difficulty of maintaining a consistent quality of performance of complex assays, led us to investigate the complete automation of these assays. We have assessed the Kemble Star 700 Automated Liquid Handling system in the performance over 1 year of several serological tests (CFTs, anti-streptolysin O titre, Venereal Disease Reference Laboratory micro-agglutination test, Treponema pallidum Haemagglutination test and ELISA). This equipment is capable of automated sampling, serial dilution, reagent addition and plate washing. These activities are controlled by user-defined protocols on a microcomputer which, once initiated, can be performed without the presence of technical staff. Protocols are generated by use of the menu-driven application software supplied with the machine. Protocols are stored on hard disk from which they can

Received 14 Nov. 1990; accepted 31 Jan. 1991

* Kemble Instrument Company Limited, Marchants Way, Burgess Hill, Sussex. be recalled to run assays repetitively or for further amendment if necessary.

\section{Materials and methods}

\section{Equipment configuration}

The Star 700 processor comprises a robotic arm which incorporates three linear motors that permit $\mathrm{x}$, $y$ and vertical (2) movement over a work-platform on which racks and containęrs can be located. Various probes, tubes, racks and reagent containers may be used. The configuration that we used had a dual probe of two PTFE-coated stainless steel sampling nozzles and a four-nozzle plate washer attached to the robotic arm. The liquid column in each nozzle is controlled independently by a separate piston pump and, in the assays, each pump was primed with diluent fluid. Probes are fitted with liquid level sensing circuitry that allows the probe to detect the surface of samples by measuring conductivity. The probe can be programmed to move down at the same rate as the liquid surface during aspiration operations which minimises the external contamination of the probe and allows the automatic calculation of the remaining volume of fluid in a container. Warnings are issued to the microcomputer screen if the volume in the container is insufficient. An "active" (aspiration) system for washing the probe to prevent carry-over is fitted to the instrument. Used wash solution is removed from the base of the wash bowl to a closed waste bottle by a vacuum system. After delivering a sample or reagent, the probe is located over the plastic washbowl and diluent is dispensed through the probe. The outside of the probe is washed by a stream of wash fluid delivered by pressurised air to the washbowl. The volume of 
diluent delivered for washing and the stage at which this operates can be programmed.

For washing microtitration plates, wash solution is delivered from a pressurised vessel through the fournozzle head to the wells and the waste is aspirated to an evacuated container. Protocols may be set to allow wells to soak in wash solution for a period of time before aspiration. The protocol also specifies the number of times the wells are filled and aspirated and the length of time this is to take.

\section{Computer protocols}

Computer protocols were built up in stages by means of the menu-driven software package, "Progen", developed by the Kemble Instrument Company. The software was used to build up command sequences that gave the names of "areas" on the work-platform to which probes were to be moved, the volumes to be aspirated or dispensed, and details of air gaps between fluids, pump speeds etc. For air gaps and pump speeds, the program supplied default settings which could be altered in the computer. For other variables, prompts were issued that gave the permitted ranges. Complex protocols were easy to set up and modify.

Before a protocol can be run, the $\mathrm{x}, \mathrm{y}$ and $\mathrm{z}$ coordinates of the various racks and containers must be logged on the computer. This is achieved by use of a tracker ball unit to move the robotic arm over the working surface and the rack definition program to record measurement of the $x, y$ and vertical positions for each rack and container used. These measurements are stored for future use. The co-ordinates thus defined may be shared by any number of assay protocols which use the same racks or containers in these specified positions.

\section{Sample and reagent addition}

Sera or reagents may be aspirated or delivered separately from both nozzles of the dual probe to or from any location within the work area. The protocol can control the insertion of an air bubble between different fluids sequentially aspirated to prevent mixing within the probe. For some protocols, duplicate samples are aspirated simultaneously in both sampling nozzles and separately delivered to different reaction wells or tubes, speeding up the process by reducing the need for movement across the work platform.

Reagent addition is handled in two ways; "dip and sip" or "primed through" addition. With "dip and sip", reagent is aspirated by the probe from a container on the work area in the same way as for sampling. Reagents can be taken up, an air bubble inserted and the sample then aspirated; both may be dispensed in a single operation. In "primed through" additions the piston pumps are primed with reagent rather than diluent through tubing that dips into a reagent reservoir placed on the work-platform. This allows reagent to be rapidly dispensed to a large number of locations without the need for the probe to return to a container to aspirate more fluid.

Heat-labile reagents may be affected by the dispensing method chosen. After initial problems with loss of complement activity, thermocouples were used to monitor the temperature of complement as it passed through the pumping system. Cooled complement was placed on the work platform in a small vacuum flask and samples were repeatedly dispensed into a plastic universal container, first by the "prime through" method and then by use of "dip and sip" techniques. Thermocouple probes were placed in the cooled complement reservoir, in the receiving vessel and were also attached to the metal components of the pumps to monitor temperatures during these operations.

\section{Accuracy and precision}

The precision of "primed through" dispensing was assessed by repeated dispensing of a set volume of water into an empty container and weighing the expressed volume. This was repeated after an interval of 15 months routine use to determine if mechanical wear had any effect on accuracy. To determine the variance of dispensing, 24 aliquots of $100 \mu$ l of water were dispensed sequentially from each of the two nozzles into individual pre-weighed test tubes which were then re-weighed to determine the volume of each aliquot dispensed. Precision during "dip and sip" operations was assessed in a similar way with both serum and an aqueous buffer. The expected dispensed weight was determined by weighing accurately measured $100-\mathrm{ml}$ volumes of serum and buffer in grade A volumetric glassware. Precision in double dilution series was measured by double diluting $100-\mu$ l volumes of aqueous toluidine blue $0.015 \%$ solution. The dilution series was assessed by reading the optical density of dilutions on a spectrophotometer at $570 \mathrm{~nm}$.

\section{Assays}

CFT's were performed by the method recommended by Bradstreet and Taylor ${ }^{1}$ modified for microtitrationplate methodology with four volumes in microtitration plates with a standard volume of $25 \mu \mathrm{l}$. Each serum was diluted manually to 1 in 8 and inactivated at $56^{\circ} \mathrm{C}$ for $30 \mathrm{~min}$. The dilutions were titrated in eight rows of a microtitration plate across six columns, i.e., two sera were assayed on each plate. Known high-titre sera were titrated across 12 columns. A different antigen was added to each row. Complement was used at $3 \mathrm{HD} 50$ (50\% haemolytic dose) and stored in a small vacuum flask. Microtitration plates were incubated at $4^{\circ} \mathrm{C}$ overnight. The next day plates and sensitised erythrocytes were warmed to $37^{\circ} \mathrm{C}$ for $30 \mathrm{~min}$, and the sensitised red cells were then added to the plates. Incubation was continued for a further $30 \mathrm{~min}$ with agitation for $1 \mathrm{~min}$ after $15 \mathrm{~min}$ and again at the end. Plates were centrifuged at $1500 \mathrm{~g}$ for $4 \mathrm{~min}$ before reading. 
When a Microcompupet eight-channel peristaltic pump was used, titrations required the initial addition of $50 \mu \mathrm{l}$ of diluted serum to the first column of each row with a hand-held micropipette. After priming the Microcompupet with CFT buffer, doubling dilutions were prepared along eight rows at a time. When all the titrations were completed the Microcompupet was primed with a different antigen in each channel, allowing eight antigens to be added at a time.

With the Star 700, the diluted and inactivated sera for titration were placed in a 96-place carousel. Antigens contained in plastic $28-\mathrm{ml}$ containers were placed on the work-platform in a standard laboratory rack. The locating template supplied with the equipment and designed to hold six microtitration plates was used to hold plates accurately in position. The probe was primed with CFT buffer. The machine was programmed to perform titrations by diluting the serum and adding the antigen in a single manoeuvre whilst the two-nozzle probe was over a well. The probe picked up $210 \mu \mathrm{l}$ of diluted serum in each of the two nozzles and delivered $50 \mu \mathrm{l}$ into the eight wells of the first column of the microtitration plate. The probe was washed by means of the active washbowl to prevent carry-over. Sufficient antigen for addition to all six columns of a row was then picked up in nozzle 1 . Nozzle 2 was used to make doubling dilutions as the probe moved along the row while antigen was added to each well from nozzle 1 . The pick-up point of each dilution was at a fixed height from the bottom of the well. Complement was added to the plates, after all dilutions and antigen additions had been completed, by a separate protocol which picked up complement in both nozzles by "dip and sip".

The ELISA method assessed was that developed for aspergillus serology by Trull et al. ${ }^{2}$ Two microtitration trays were coated overnight with either aspergillus somatic antigen or aspergillus culture filtrate antigen (Bencard). Sera were tested at a dilution of 1 in 100 in duplicate against each antigen. Antibody binding was detected with goat anti-human IgG conjugated to alkaline phosphatase. Distributed controls were used to control variation across the plate.

For the automated test, two protocols were defined. In the first, $100-\mu 1$ volumes of each of the two antigens were dispensed in a single stage to flexible microtitration plates which were incubated overnight at $37^{\circ} \mathrm{C}$ to allow the antigen to coat the plates. The following day, with the second protocol, the four-nozzle wash probe washed the plates three times with a 4-min soak between washes two and three followed by a complete aspiration of wash fluid from the plate. Ten $\mu$ l of test or control serum was then aspirated in one nozzle of the dual probe and washed into a tube with $450 \mu \mathrm{l}$ of diluent from the same nozzle, together with $500 \mu \mathrm{l}$ of diluent from the other nozzle, to give an initial dilution of 1 in 100. Each step was followed by a wash to waste of $750 \mu$ of diluent from each nozzle. The dual nozzle sample probe was then used to dispense $100-\mu$ l volumes of the diluted sera in duplicate across the microtitration plates; the duplicates were dispensed in adjacent columns. Negative and positive control sera were dispensed into set positions across the plate to provide controls for plate variation.

After this dilution and dispensing, the microtitration plates were removed from the work-platform and incubated in a moist box at $37^{\circ} \mathrm{C}$ for $1 \mathrm{~h}$ while the conjugate was prepared. Plates were returned to the same locating template on the work-platform, washed as before and $100-\mu 1$ volumes of conjugate were added to wells. The protocol was set up with counters and conditional loops that allowed reagent to be added only to those wells to which test sera or controls had been added. After a further incubation stage, plates were once more returned to the work-platform. First, one plate was washed and $100-\mu l$ volumes of substrate were added, at which point the protocol permitted a user-controlled pause to allow the plate to be removed to a spectrophotometer for the reaction rate to be monitored. Once the optical density of the positive control had reached 0.800 the plate was returned to the work-platform and the protocol resumed, adding $100 \mu \mathrm{l}$ of $3 \mathrm{M} \mathrm{NaOH}$ to test and control wells to stop the reactions. The protocol repeated this substrate addition stage for plate 2. Although the robot was inactive during incubation stages, it would have been possible to interrupt the protocol temporarily at that point and run another test. On recalling the original protocol it would have resumed from the point at which it was suspended.

\section{Results}

\section{Fluid sensing}

The manufacturer's claim that volumes as low as $50 \mu \mathrm{l}$ can be detected by the conductivity sensor if rack parameters are correctly defined was evaluated. In serial dilutions with $50-\mu$ l volumes in microtitration trays, we have found that the equipment failed to detect fluid in approximately $5 \%$ of wells, which resulted in the suspension of further dilution of that sample. This problem was overcome by turning off liquid-level sensing when plates were being used and setting a fixed aspiration height for the probe within the protocol which would always be below the fluid surface. No such problems were encountered with volumes above $100 \mu \mathrm{l}$.

\section{Reagent addition}

The "primed through" mode was tried initially for adding complement in CFT's and it was found that a higher concentration of complement had to be used than was required with manual methods. Temperature monitoring with a thermocouple demonstrated a rise of $4.5^{\circ} \mathrm{C}$ as fluid passed through the pumps set at the default speeds; this was thought to be inactivating the complement. With "dip and sip" addition, the rise 
was $1.5^{\circ} \mathrm{C}$ and there was no loss of complement activity. In "dip and sip" addition, a thermocouple in the receiving vessel recorded a slight, momentary fall in temperature as each aliquot of complement was dispensed, showing that the fluid in the probe was cooler than that to which it was being added. This was the mode adopted for further tests.

\section{Plate washing}

For the ELISA assay two initial washes, followed by a 4-min soak and a final wash, were found to be adequate.

\section{Precision of dispensed volumes}

When 100 aliquots were dispensed with the pump set at default speeds, the imprecision of the volumes delivered varied with the volume being dispensed. The total error when dispensing $10025-\mu 1$ volumes was $-2.0 \%$ with a mean volume of $24.5 \mu \mathrm{l}$. For 100 $100-\mu \mathrm{l}$ volumes, the total error was $-0.2 \%$ with a mean volume of $98.8 \mu \mathrm{l}$. The larger discrepancy with the small volume did not create difficulties in CFTs, but it should be borne in mind in situations where precise work is important. Use of a slower pump speed could reduce the error. Examination of the performance of each nozzle of the dual probe separately with $100-\mu l$ volumes revealed a mean percentage error per aliquot of $-0.3 \%$ for nozzle 1 and $-0.2 \%$ for nozzle 2. When re-examined after routine use for 15 months, the volumes dispensed by nozzle 1 showed the same error of $-0.3 \%$ with $100-\mu$ l volumes, but the mean error with nozzle 2 had increased to $-0.6 \%$. The mean volume dispensed was $99.7 \mu \mathrm{l}$ (coefficient of variation [cv] $0.8 \%$ ) for nozzle 1 and $99.4 \mu \mathrm{l}$ (cv $0.7 \%$ ) for nozzle 2.

With "dip and sip" sampling, there was no difference in volume error when serum and aqueous solutions were used. The total error with $100100-\mu 1$ aliquots was $+1.9 \%$ and with $25-\mu$ l aliquots $+6.9 \%$. The mean excess volume dispensed was similar for each volume size- -1.9 and $1.7 \mu$ respectively.

\section{Precision of double dilutions}

Replicate series of dilutions of toluidine blue showed excellent agreement up to a dilution of 1 in 128. Above this, the toluidine blue was too dilute to produce a change in optical density readings. Precision was superior to that of replicate series diluted with the Microcompupet manual method (fig. 1). Replicates of sera titrated in parallel by complement fixation on the sample processor also gave the same result. Over 500 CFT titrations were performed with 12 different antigens in parallel by the manual method and the automated sample processor. The results obtained with the processor compared favourably with those of the manual method. A close comparison of 65 sera titrated in parallel by manual and robotic methods (fig. 2) showed that the results obtained with all but four sera were within half of a two-fold dilution of each other. Of the other four sera, three gave titres which were two-fold different and one a titre that was four-fold different.

\section{Carry-over}

Carry-over when sampling of a strongly positive serum was followed by a negative serum was not detected in the negative well in the ELISA or CFT assays used.

\section{Materials consumed}

More reagent and diluent were used in tests done with the sample processor than when the manual method was used. To maintain precision a 5-10 $\mu$ l

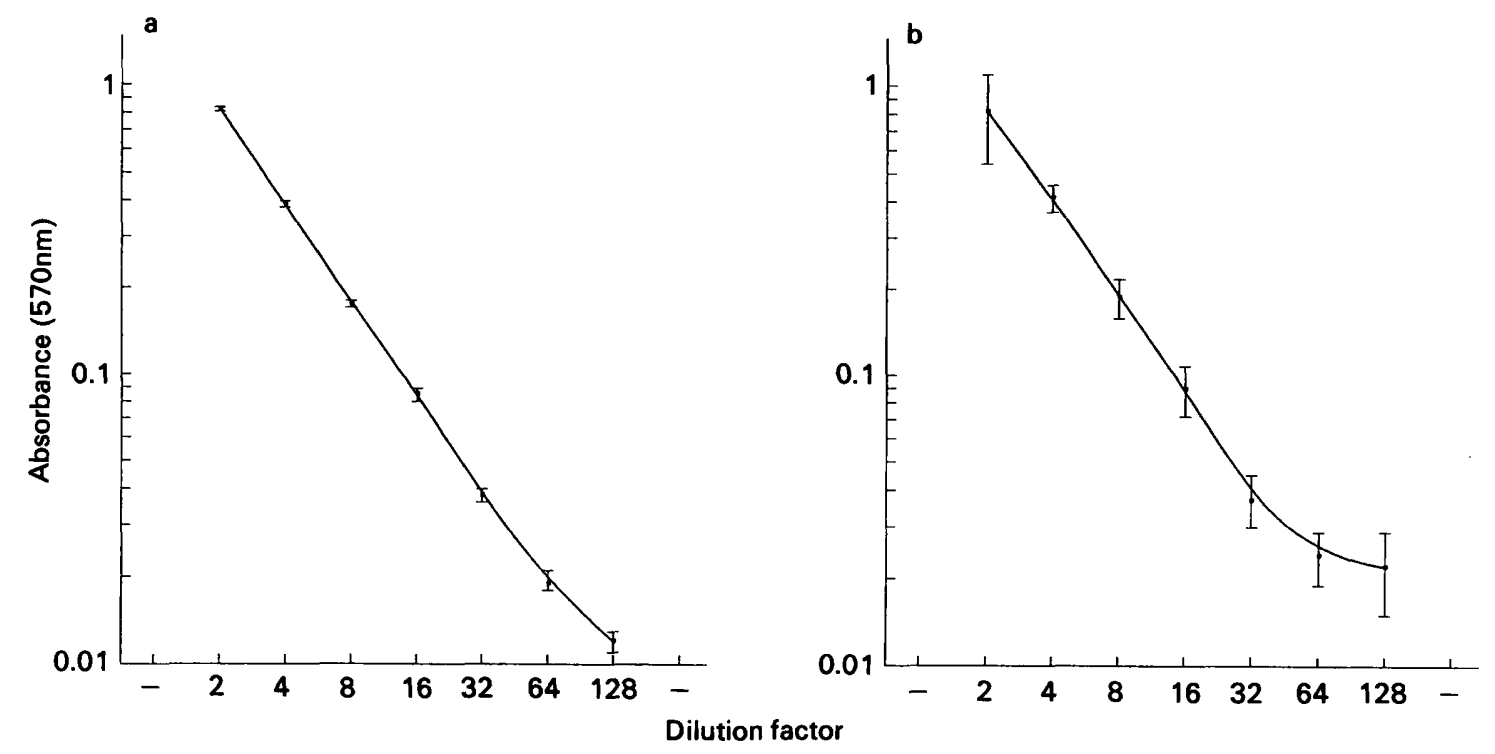

Fig. 1. Comparison of dilution series across a microtitration plate (mean absorbance and SD) by (a) automated and (b) manual methods. 


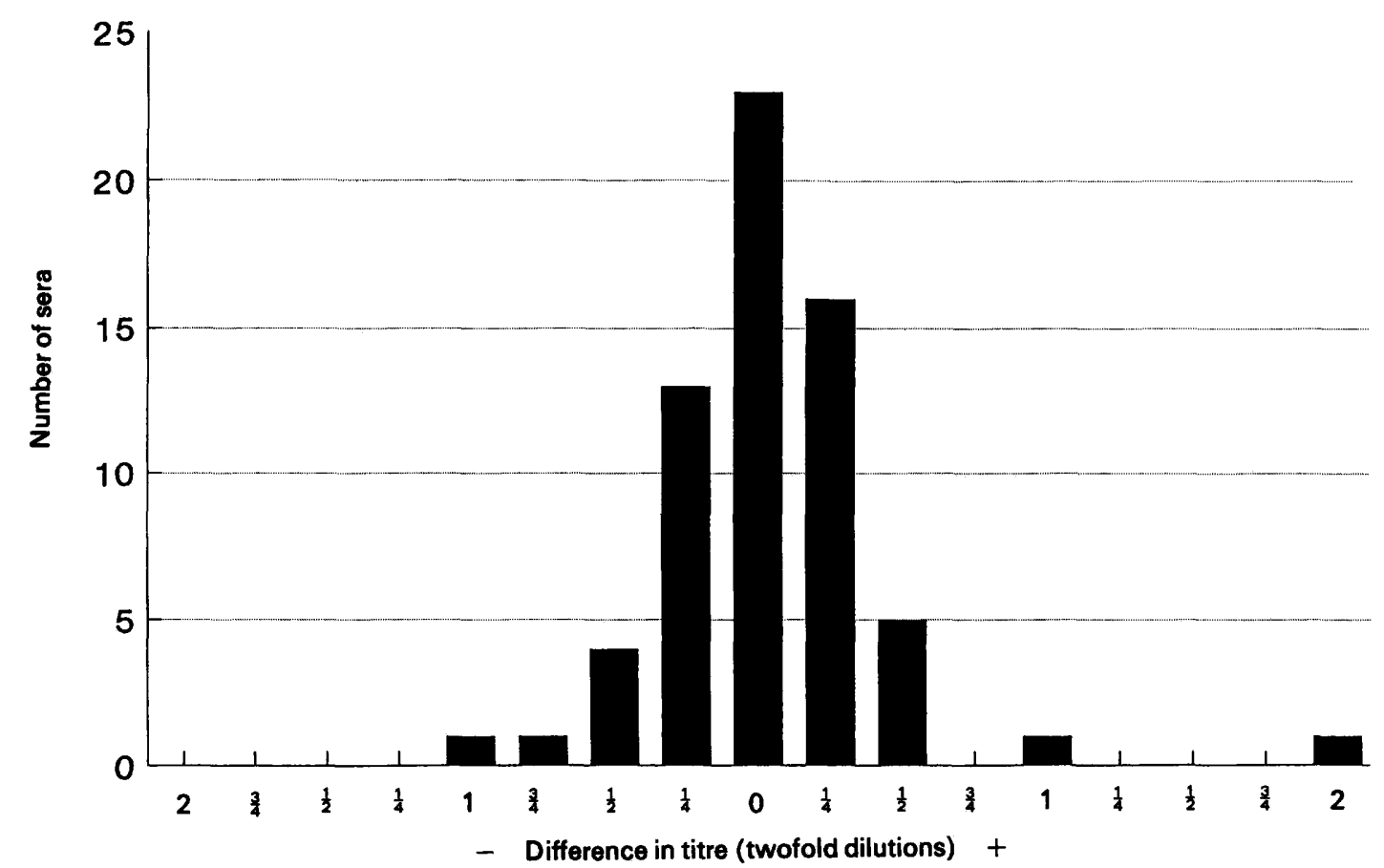

Fig. 2. Differences between CFT titres of sera assayed in parallel by manual and automated methods.

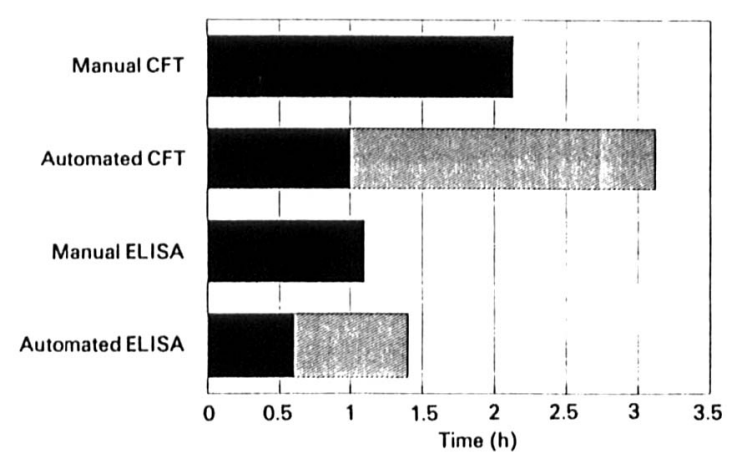

Fig. 3. Comparison of time taken to perform manual and automated assays, technologist in attendance, $\mathbb{Z}$ unattended machine.

excess of reagent was picked up by the probe in "dip and sip" operations. This was washed to waste. In CFTs and ELISA, twice the amount of diluent used in the manual method was required with the processor to prime pumps and wash the probes between samples and reagents. This is important if commercial serological kits are to be used with the automated system because manufacturers of kits may have to be asked to supply additional diluent.

\section{Technical time}

Because the robotic system addresses only a single well at a time whereas multichannel pipettes are used in manual methods, the time taken to complete a batch of CFTs was c. $30 \%$ longer (fig. 3), but the time staff spent at the bench was reduced considerably (table I). Closer analysis showed that most of the technical time required for assays by the robotic system was used at the beginning for preparation of samples and reagents. Thereafter, the processor ran unattended for $30-\mathrm{min}$ periods during the titration and antigen-addition stages. The initial dilution of sera before heat inactivation for CFTs has not yet been automated but this could provide further scope for time saving.

Similarly, with the ELISA assay, the use of 12channel micropipettes and a plate washer in the manual assay, compared with a single probe and fournozzle wash head of the processor meant that the total time to complete the assay was $20 \%$ longer for the automated assay (fig. 3). However, there was still a saving of technical time in the sample dilution and distribution stage when single-channel pipetting was used in both assays (table II). The automated assay also eliminated the possibility of samples and controls being added to the wrong wells. The optical density readings obtained from assay to assay with the controls was much more consistent with the automated assay.

\section{Discussion}

The Kemble Star 700 automatic processor can be programmed satisfactorily to perform CFTs and ELISA. The time to perform some operations was approximately 2.5 times longer than that taken by an experienced technologist using multi-channel pipettes (10 min vs $4 \mathrm{~min}$ per microtitration plate for a CFT titration). However, the automated tests were always completed during the working day and the technologist was released to other duties for prolonged periods during machine operation. With ELISA, only $38 \mathrm{~min}$ of worker's time was required to process 18 sera in duplicate with two antigens. With CFTs, it was 
Table I. Comparison of time taken to perform CFT with 40 sera against eight antigens

\begin{tabular}{l|rlrl}
\hline & \multicolumn{4}{c}{ Time (min) required for } \\
\cline { 2 - 4 } Operation & \multicolumn{3}{|c}{ automated assay } & manual assay \\
\cline { 2 - 4 } & operator & machine & (total) \\
\hline $\begin{array}{l}\text { Sample and reagent } \\
\text { preparation }\end{array}$ & 39 & 0 & $(39)$ & 39 \\
$\begin{array}{l}\text { Titration and antigen } \\
\text { addition }\end{array}$ & 7 & $3 \times 30^{*}$ & $(97)$ & 69 \\
$\begin{array}{l}\text { Complement addition } \\
\text { Maintenance and } \\
\text { manual additions }\end{array}$ & 9 & $3 \times 8^{*}$ & $(33)$ & 10 \\
$\quad$ Totals & 5 & 0 & $(5)$ & 3 \\
\hline
\end{tabular}

* Machine protocol handles plates in batches of six and prompts operator to put next set in place on work platform during these stages.

Table II. Comparison of time taken to perform ELISA with 18 sera against two antigens

\begin{tabular}{|c|c|c|c|c|}
\hline \multirow{3}{*}{ Operation } & \multicolumn{4}{|c|}{ Time ( $\mathrm{min}$ ) required for } \\
\hline & \multicolumn{3}{|c|}{ automated assay } & \multirow{2}{*}{ manual assay } \\
\hline & operator & machine & (total) & \\
\hline $\begin{array}{l}\text { Sample and reagent } \\
\text { preparation } \\
\text { Plate wash, sample } \\
\text { dilution and dispensing } \\
\text { Incubation } \\
\text { Wash two plates and add } \\
\text { conjugate } \\
\text { Incubation } \\
\text { Wash plate } 1 \text { and add } \\
\text { substrate } \\
\text { Monitor OD and add } \\
\text { stop solution } \\
\text { Wash plate } 2 \text { and add } \\
\text { substrate } \\
\text { Monitor OD and add } \\
\text { stop solution } \\
\text { Totals }\end{array}$ & $\begin{array}{r}15 \\
5 \\
2 \\
2 \\
2 \\
5 \\
3 \\
1 \\
3 \\
38\end{array}$ & $\begin{array}{r}0 \\
15 \\
0 \\
10 \\
0 \\
8 \\
3 \\
\\
8 \\
3 \\
47\end{array}$ & $\begin{array}{r}(15) \\
(20) \\
(2) \\
(12) \\
(2) \\
(13) \\
(6) \\
(9) \\
(6) \\
(85)\end{array}$ & $\begin{array}{r}15 \\
20 \\
2 \\
7 \\
2 \\
6 \\
4 \\
6 \\
6 \\
4 \\
66\end{array}$ \\
\hline
\end{tabular}

possible to titrate 60 sera against eight antigens within a working day. Manual CFT methods would occupy an experienced technologist for the whole day. The processor reduced this to half a day of technologist time. Staff operating the processor have found the processing of large numbers of serological tests less stressful than when manual methods were used. CFTs and ELISA are some of the most complex tests performed in microbiology laboratories. That it has been possible to program the Star 700 processor satisfactorily indicates that there should not be difficulty with the manipulations required for other tests. ELISA are technically demanding and require practice to obtain consistent results. With the Star 700 processor, staff with no previous experience of ELISA techniques have been trained in a very short time to produce acceptable results.

\section{References}

1. Bradstreet CMP, Taylor CED. Technique of complementfixation test applicable to the diagnosis of virus disease. Monthly Bulletin of the Ministry of Health 1962; 21 : 96-104.

2. Trull AK, Parker J, Warren RE. IgG enzyme linked immunosorbent assay for diagnosis of invasive aspergillosis: retrospective study over 15 years of transplant recipients. J Clin Pathol 1985; 38: 1045-1051. 